\title{
Overestimation of Carotid Artery Stenosis by Carotid Duplex Ultrasonography Due to Contralateral Occlusion
}

\author{
Hee Jo Han, MD* (D); Yunjung Choi, MD* iD; Yo Han Jung, MD, PhD (D); Kyung-Yul Lee, MD, PhD ${ }^{*} \dagger$ (iD \\ Department of Neurology, Gangnam Severance Hospital, Yonsei University College of Medicine*, Seoul; Severance Institute for Vascular and \\ Metabolic Research, Yonsei University College of Medicine ${ }^{\dagger}$, Seoul, Korea
}

Carotid duplex ultrasonography is a noninvasive and economical method used to diagnose and monitor carotid artery diseases. However, the accuracy of carotid duplex ultrasonography is decreased in the presence of contralateral high-grade stenosis or occlusion. Among the criteria for evaluating the degree of carotid artery stenosis, the Society of Radiologists in Ultrasound consensus criteria are the most widely used owing to their high sensitivity and specificity. Here, we have presented a case in which internal carotid artery (ICA) stenosis was overestimated using the consensus criteria due to the chronic occlusion of the contralateral ICA.

J Neurosonol Neuroimag 2020;12(2):73-75

Key Words: Carotid stenosis; Ultrasonography, Doppler, duplex; Cerebral infarction

\author{
Received: July 9, 2020 \\ Revised: August 19, 2020 \\ Accepted: August 19, 2020 \\ Correspondence: \\ Kyung-Yul Lee, MD, PhD \\ Department of Neurology, \\ Gangnam Severance Hospi- \\ tal, Yonsei University College \\ of Medicine, 211 Eonju-ro, \\ Gangnam-gu, Seoul o6273, \\ Korea \\ Tel: +82-2-2019-3325 \\ Fax: +82-2-3462-5904 \\ E-mail: kylee@yuhs.ac
}

Carotid duplex ultrasonography is a noninvasive and economical method used to diagnose and monitor carotid artery diseases. The Society of Radiologists in Ultrasound consensus criteria, the most widely used criteria for evaluating the degree of internal carotid artery (ICA) stenosis, stratifies ICA stenosis into several degrees. ${ }^{1}$ Among the several parameters, peak systolic velocity (PSV) and presence of plaque are primarily used. According to the consensus criteria, severe stenosis (>70\% stenosis to near occlusion) is diagnosed when ICA PSV is $>230 \mathrm{~cm} / \mathrm{s}$ and visible plaque and luminal narrowing are observed. Severe stenosis should be carefully diagnosed because overestimation can lead to unnecessary surgical treatment or stenting. Here, we have reported a case in which ICA stenosis was overestimated by carotid duplex ultrasonography based on the consensus criteria due to the chronic occlusion of the contralateral ICA.

\section{CASE}

An 83-year-old man presented with a sudden occurrence of dysarthria. On neurological examination, he had mild dysarthria and right facial palsy. He had a National Institutes of Health Stroke Scale score of 2. He had history of diabetes mellitus and cerebellar infarction without sequelae. Previous magnetic resonance angiography had revealed moderate stenosis of the left ICA. He was administered aspirin, clopidogrel, and rosuvastatin $10 \mathrm{mg}$ for carotid artery stenosis. Initial brain computed tomography angiography (CTA) showed complete occlusion of the left ICA and moderate stenosis of the right ICA (Fig. 1A). Diffusion-weighted magnetic resonance imaging revealed acute infarction in the left middle cerebral artery territory (Fig. 1B), and perfusion-weighted imaging showed slight perfusion delay in the left hemisphere. Further evaluation using digital subtraction angiography (DSA) revealed that his left proximal ICA was occluded, and collateral circulations via anterior and posterior communicating arteries supplied blood to the left hemisphere (Fig. 1C). 
Carotid duplex ultrasonography, performed 3 years after the cerebral infarction, revealed a very high PSV of the right ICA $(329.7 \mathrm{~cm} / \mathrm{s})$ (Fig. 2A). Therefore, carotid artery stenting was considered for suspected severe stenosis at the right proximal ICA. However, DSA showed only moderate stenosis in the right proximal ICA (Fig. 2B). Therefore, previous medical treatment was continued without carotid artery stenting.

\section{DISCUSSION}

In October 2002, the Society of Radiologists in Ultrasound established the consensus criteria for the diagnosis of ICA stenosis. According to the consensus criteria, a cutoff value of PSV for diagnosing $50 \%$ and $70 \%$ ICA stenosis is 125 and $230 \mathrm{~cm} / \mathrm{s}$, respectively. ${ }^{1}$ Since the establishment of the consensus criteria, several studies have been conducted to validate the criteria; most studies have showed favorable results. AbuRahma et al. ${ }^{2}$
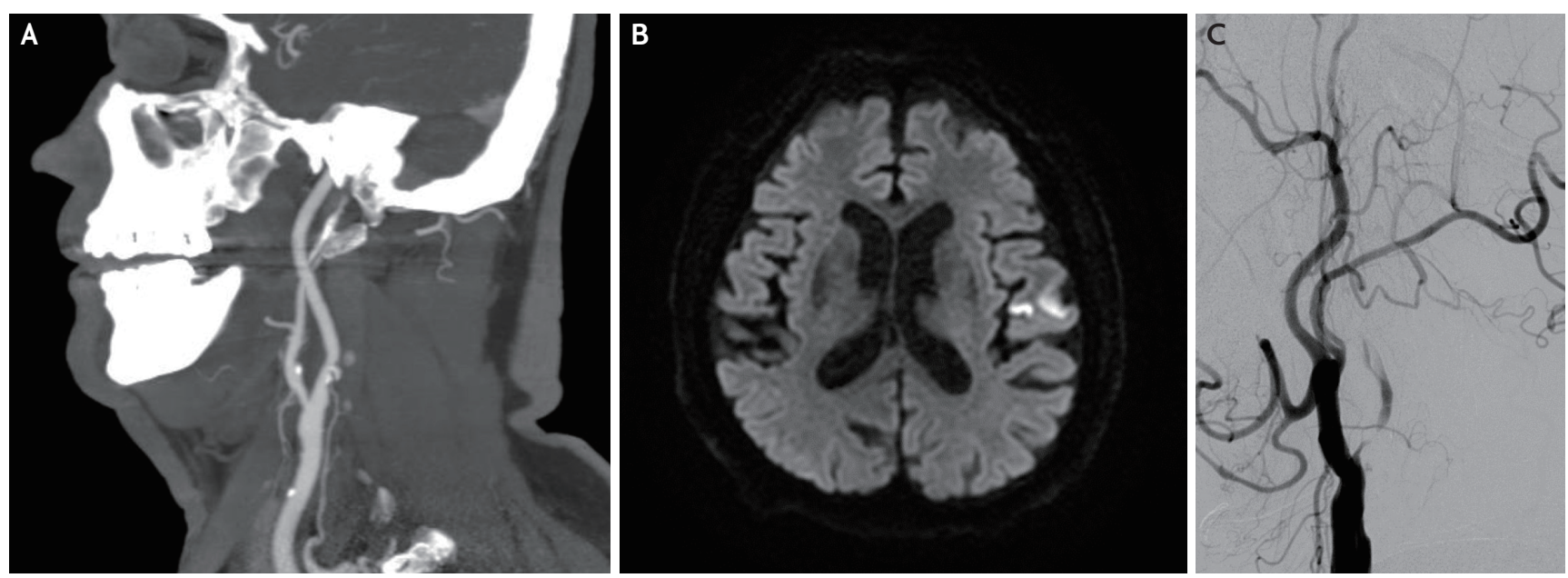

FIG. 1. Initial computed tomography angiography (CTA), diffusion-weighted imaging, and digital subtraction angiography. (A) CTA shows moderate stenosis of the right internal carotid artery. (B) Diffusion-weighted magnetic resonance imaging shows acute infarction in the left middle cerebral artery territory. (C) Digital subtraction angiography shows complete occlusion of the left proximal internal carotid artery.
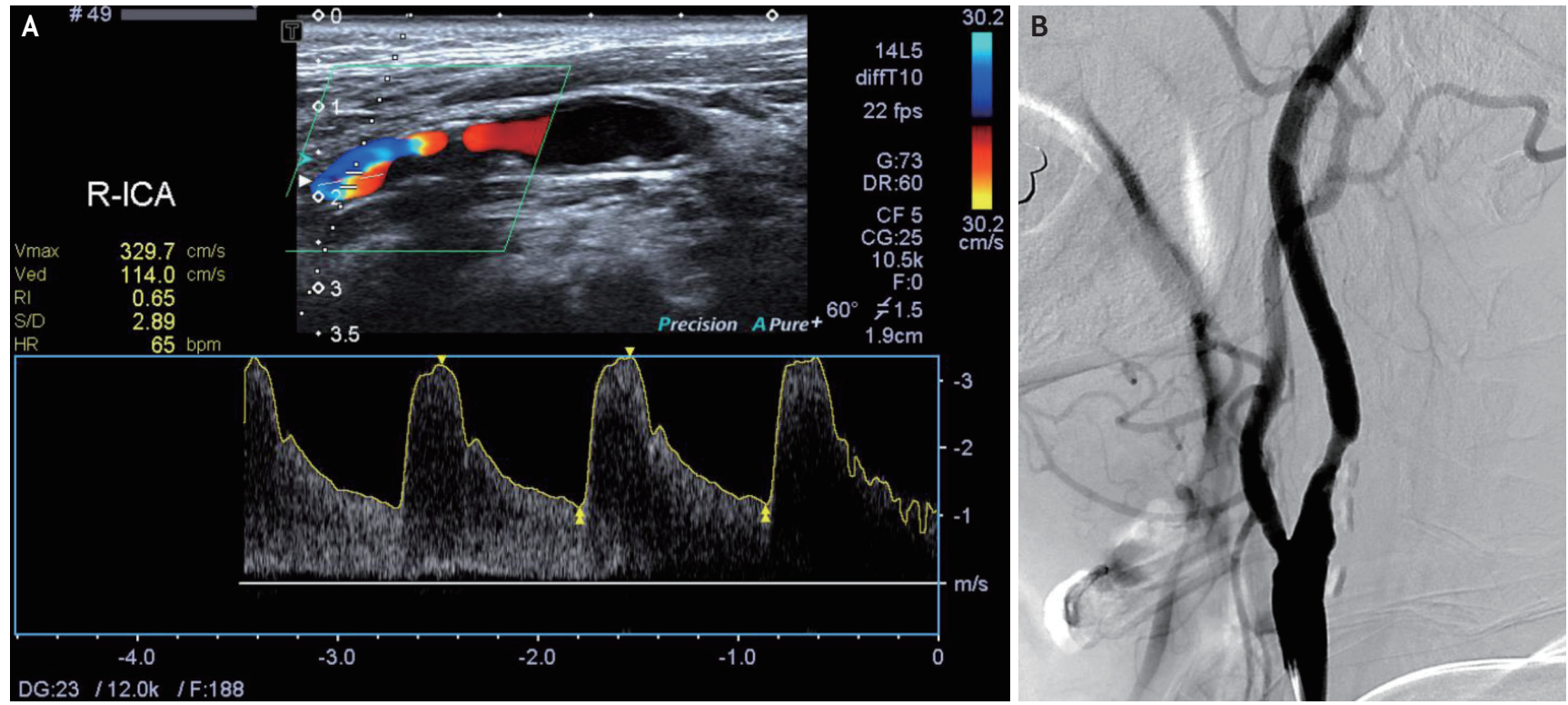

FIG. 2. Carotid duplex ultrasonography and digital subtraction angiography (DSA) of the right internal carotid artery. (A) Carotid duplex ultrasonography shows very high peak systolic velocity $(329.7 \mathrm{~cm} / \mathrm{s})$. (B) DSA shows moderate stenosis in the right proximal internal carotid artery. 
concluded that a PSV of $\geq 230 \mathrm{~cm} / \mathrm{s}$ for $\geq 70 \%$ stenosis has a sensitivity of $99 \%$, specificity of $86 \%$, and overall accuracy of $95 \%$. According to these studies, carotid duplex ultrasonography is a suitable diagnostic method to evaluate carotid artery diseases because of its high sensitivity and specificity.

However, the accuracy of carotid duplex ultrasonography is decreased in the presence of contralateral highgrade stenosis or occlusion. Carotid artery stenosis has been overestimated by carotid duplex ultrasonography based on the conventional criteria due to contralateral carotid artery disease in several cases..$^{3-5}$ Decreased PSV after contralateral carotid endarterectomy has already been reported previously; this can be considered evidence of overestimation. ${ }^{5,6}$

This overestimation possibly occurs due to remodeling of the brain blood flow distribution. In the normal vascular structure, the right and left ipsilateral ICAs supply blood to the right and left cerebral hemisphere, respectively. However, in the vascular structure of a unilateral chronic ICA occlusion, the nonocclusive ICA supplies blood to both the cerebral hemispheres. The blood volume flow through the nonocclusive ICA increases to supply enough blood to both the hemispheres through collateral pathways, which in turn increases, the blood flow velocity. ${ }^{7}$ Van Everdingen et al. ${ }^{3}$ have observed increased blood volume flow through the nonocclusive ICA in patients with symptomatic ICA stenosis or occlusion using magnetic resonance flow quantification. They have reported that increased blood volume flow through the ICA frequently causes an increased velocity, which can be measured using carotid duplex ultrasonography. ${ }^{3}$

Our case is an example of ICA stenosis overestimation by carotid duplex ultrasonography due to contralateral ICA occlusion. The patient had chronic occlusion at the left ICA with collateral circulations via the anterior and posterior communicating arteries. Carotid duplex ultrasonography showed a relatively higher degree of stenosis than the actual degree of stenosis based on the PSV in the nonocclusive ICA.

In conclusion, when evaluating ipsilateral ICA in patients with contralateral ICA occlusion using carotid duplex ultrasonography, the possibility of overestimation should always be considered. To prevent unnecessary intervention, comprehensive assessment using other diagnostic tools such as CTA or magnetic resonance angiography is required.

\section{Conflicts of Interest}

No potential conflicts of interest relevant to this article was reported.

\section{REFERENCES}

1. Grant EG, Benson CB, Moneta GL, Alexandrov AV, Baker JD, Bluth EI, et al. Carotid artery stenosis: grayscale and Doppler ultrasound diagnosis--Society of Radiologists in Ultrasound consensus conference. Ultrasound Q. 2003;19:190-198.

2. AbuRahma AF, Srivastava M, Stone PA, Mousa AY, Jain A, Dean LS, et al. Critical appraisal of the carotid duplex consensus criteria in the diagnosis of carotid artery stenosis. J Vasc Surg. 2011;53:53-59; discussion 59-60.

3. van Everdingen KJ, van der Grond J, Kappelle LJ. Overestimation of a stenosis in the internal carotid artery by duplex sonography caused by an increase in volume flow. J Vasc Surg. 1998;27:479-485.

4. Preiss JE, Itum DS, Reeves JG, Duwaryi Y, Rajani R, Veeraswamy R, et al. Carotid duplex criteria for patients with contralateral occlusion. J Surg Res. 2015;193:28-32.

5. Ray SA, Lockhart SJ, Dourado R, Irvine AT, Burnand KG. Effect of contralateral disease on duplex measurements of internal carotid artery stenosis. Brit J Surg. 2000;87:10571062.

6. Kolkert JL, van den Dungen JJ, Loonstra J, Tielliu IF, Verhoeven EL, Beck AW, et al. Overestimation of contralateral internal carotid artery stenosis before ipsilateral surgical endarterectomy. Eur J Radiol. 2011;77:68-72.

7. van Everdingen KJ, Klijn CJ, Kappelle LJ, Mali WP, van der Grond J. MRA flow quantification in patients with a symptomatic internal carotid artery occlusion. The Dutch EC-IC Bypass Study Group. Stroke. 1997;28:1595-1600. 